\title{
FDG PET and cognitive symptoms of dementia
}

\author{
Daniela Perani
}

Received: 13 May 2013/Accepted: 8 July 2013/Published online: 30 July 2013

(C) Italian Association of Nuclear Medicine and Molecular Imaging 2013

\begin{abstract}
The fundamentals of FDG PET are well established, and are based on extensively explored molecular mechanisms. The existing methods for semiquantitative assessment of brain functional changes provide topographical maps of molecular alterations both for group and single-subject comparisons. This functional brain-based approach to derangement of high-order cognition has provided and will continue to provide highquality findings that will help further understanding of brain cognitive activity and of the brain-cognition correlates and their importance in the diagnosis of the underlying pathology. This article examines the relationship between changes in brain metabolic activity and the resulting impairment of high-order cognition in dementia conditions. FDG PET imaging in subjects with underlying neurodegenerative pathology has emerged as a crucial tool available to the fields of cognitive neurology and neuroscience for both diagnosis and evaluation of the involved neural systems, extending the neuropsychology-based understanding of the brain/cognition relationship. As an effect of the multiple underlying pathologies, the cognitive deficits encountered encompass different aspects of neuropsychology: language, memory, performance monitoring, decision making, reasoning, and even "personality." It is to be noted that FDG PET studies of brain cognitive reserve have so far supported the existence of functional reserve phenomena in neurodegenerative dementia, and specifically in AD. The newly integrated PET/MRI techniques will likely become crucial clinical and research
\end{abstract}

\section{Perani $(\bowtie)$}

Division of Neuroscience, Nuclear Medicine Department,

San Raffaele Scientific Institute, Vita-Salute San Raffaele

University, Via Olgettina 60, 20132 Milan, Italy

e-mail: perani.daniela@hsr.it tools in neurology, nuclear medicine and in the neuroscience field generally. Hybrid PET/MRI will allow integration of the molecular information provided by PET with the various morphological and functional parameters measured by MRI, in the same subject and in a single study session. Future progress depends on exploration of these relationships to pave the way for new insights into neuroscience and neurology.

Keywords FDG PET - Cognitive decline .

Dementia $\cdot$ Diagnosis $\cdot$ Cognitive functions $\cdot$ Brain reserve

\section{Introduction}

The functional anatomy of cognition has been investigated using positron emission tomography (PET) and functional magnetic resonance imaging (fMRI) for more than 20 years. The importance of the early PET studies was far reaching. They illustrated that functional imaging could provide anatomical localization with a precision that far exceeds that attainable with human brain lesion studies. Many findings have been replicated time and time again, leading to some consistent and indisputable conclusions [1,2].

Positron emission tomography with 2-[18F]fluoro-2deoxy-D-glucose (FDG) as the tracer (FDG PET) has long been used to measure resting-state cerebral metabolic rates of glucose, a proxy for neuronal activity and a direct index of synaptic function and density [3].

The neurodegenerative dementias are a heterogeneous group of disorders, sharing a common natural history, characterized by a chronic course following a variable preclinical phase of cognitive decline, reflecting progressive neuronal dysfunction and cell loss. The clinical 
diagnosis of dementia is often made at a relatively late stage in the course of the disease, when symptoms are manifest and cognitive functions already severely affected. In addition, differential diagnosis between dementia diseases based only on clinical and cognitive assessments can be difficult in the early phase.

Selective cognitive symptoms are associated with specific alterations of functional networks and FDG PET neuroimaging studies, by defining specific brain dysfunctions, have played an important role in the neuropathological and neuropsychological research of dementia and lent substantial support to the diagnosis and differential diagnosis of dementia conditions [1]. In Alzheimer's disease (AD), for example, FDG PET studies disclosed the relationship between longterm memory deficits and hypometabolism in related neural networks including not only the hippocampal structures but also other crucial nodes such as the retrosplenial cortex and precuneus [4]. In the frontotemporal lobar degeneration (FTLD) spectrum of disorders, two major clinical presentations are classified [5]: a behavioral variant (bvFTD) and a language variant, known as primary progressive aphasia (PPA). Cerebral glucose metabolism in bvFTD subjects is mainly impaired in the mesial or dorsolateral frontal cortices, leading such impairment to be considered a specific functional marker of behavioral presentations such as apathy and disinhibition with agitation [6, 7]. FDG PET imaging in PPA also shows selective patterns of cortical hypometabolism with significant involvement of left hemispheric structures that differentiate the different aphasia syndromes [8].

As a result of research efforts aimed at the identification of in vivo biomarkers for the reliable and early detection of $\mathrm{AD}$ and other dementia pathologies, FDG PET neuroimaging, aiming to establish typical patterns of neurodegeneration, is now considered an essential part of the diagnostic algorithm [9-14].

Hypometabolic patterns typical of AD or frontotemporal dementia (FTD) have been extensively reported in large series of FDG PET studies based on semi-quantitative assessment [6, 7, 15-17]. Regional functional impairment of resting glucose metabolism has, indeed, been shown to be closely related to the severity, progression and type of cognitive impairment, thus making its assessment useful also for the diagnosis of mild cognitive impairment (MCI) due to AD [18-20]; indeed, this assessment has been shown to predict a decline from normal cognition to MCI and AD with over $80 \%$ accuracy [21]. The FTLD spectrum, including the behavioral and aphasia variants, and the neurodegenerative diseases associated with movement disorders, i.e. corticobasal degeneration and progressive supranuclear palsy, can also be better diagnosed using FDG PET [6-8, 22, 23].

Finally, FDG PET research studies have also provided fundamental support for the brain reserve hypothesis. They have shown a greater degree of impairment of regional cerebral metabolic activity for a given level of dementia in highly educated patients [24-26]. This suggests that these individuals have a functional reserve provided by their education, which masks the clinical expression of a higher degree of neurodegeneration.

On a technical level, different statistical approaches and neural network classifications have been proposed to allow better localization of regional metabolic impairment and better diagnostic accuracy in neurodegenerative and dementia conditions [15, 27-29]. In particular, the statistical parametric mapping (SPM) technique has been extensively applied to FDG PET resting (steady-state) studies with the aim of providing automated voxel-based measurements of brain hypometabolism in disease conditions. SPM-based comparisons with samples of matched normal subjects have significantly improved the study of patient groups and single cases [28, 30, 31].

Other technical aspects, in particular the availability of high-resolution scanners and advanced methods of data analysis, are also crucial factors to take into account in the evaluation of the dysfunctional brain. Hybrid PET/MRI techniques have great potential for clinical and research applications [32]. PET/MRI is of special interest for the neuroscience field, given that PET and MRI are the neuroimaging methods of choice for many clinical and scientific applications.

\section{Semi-quantitative FDG PET analysis procedures}

FDG PET scans were once used mainly for qualitative visual interpretation of images. Nowadays, however, their most widespread clinical use is in voxel-based, automated procedures for the measurement of hypometabolism.

Sensitive and specific FDG PET analysis tools for the study of brain functional impairment are crucial, particularly for the detection of early metabolic changes associated with specific cognitive symptoms. Visual interpretation of images, on the other hand, clearly lacks clear-cut milestones for distinguishing between normal and pathological scans, and only studies employing voxel-based analysis techniques (such as SPM, Neurostat, AD t-sum) can provide unbiased, statistically defined measures of brain abnormality throughout the whole brain on a voxel-by-voxel basis [1, 29, 31].

Automated tools for computer-assisted diagnosis can be categorized into two classes: voxel-based mapping tools and summary metrics of hypometabolism. Voxel-based mapping tools provide topographical estimates of the difference in metabolism compared with a dataset of normal scans on a voxel-by-voxel basis. Summary metrics are scalar numbers whose value is proportional to brain regional 
hypometabolism. Voxel-based estimates of the departure from a dataset of normal scans are summed over the volume of interest $[15,29]$. They do not provide information beyond the volume of interest. Thus, a precise map of hypometabolism in individual FDG scans is not available for clear pattern definition. On the other hand, SPM-based comparisons with samples of matched normal subjects provide maps of FDG distribution that are characterized in terms of spatial extent and peak height according to a Gaussian distribution and show where significant brain regional hypometabolism is located $[28,33,34]$.

In addition, region-of-interest (ROI) methods for FDG PET evaluation of uptake metabolism in $\mathrm{AD}$ are described in the literature and their performances in terms of computing abnormal metabolism in $\mathrm{AD}$ are well documented. In a study by Landau et al. [35], the authors reported a MetaROI approach using pre-defined regions shown, in a PubMed-based meta-analysis, to be frequently cited in FDG PET studies of AD and MCI. As a consequence of the search results, further analysis was based on a set of four regions located in the right and left angular gyri, bilateral posterior cingulate gyrus, and left middle/inferior temporal gyrus. Notably, the authors found that baseline and longitudinal FDG ROI measures were sensitive to changes both in the Alzheimer's Disease Assessment Scale-cognitive subscale and on a test of functional competence, validating the cognitive and functional relevance of longitudinal changes in FDG PET measurements.

FDG PET research and clinical studies should apply these tools since they significantly improve the detection of local dysfunctions that are also associated with behavioral and cognitive symptoms $[1,7,18]$.

What is especially relevant in this context is that FDG PET scans, in order to provide quantified measures at individual level, should be analyzed using a large normal database. However, such databases are not readily available. There are ongoing projects that aim to provide the medical and research community with validated and standardized methods and databases for research and in clinical routine (https://www.eu-decide.eu/).

With regard to the in vivo imaging biomarkers currently identified, it is worth noting that FDG PET features as a decisive tool in the recent guidelines [10-14]. The reference-based recommendations, however, also included a large body of PET imaging literature based on the use of visual methods that, greatly depending on the observer's experience, are associated with reduced sensitivity and specificity and lack a clear cut-off between normal and pathological findings. To overcome these limitations, FDG PET images should be assessed using semi-quantitative or voxel-wise analyses that increase sensitivity and specificity and allow more accurate differential diagnosis in the early phases of dementia conditions.

\section{Alzheimer's disease}

Alzheimer's disease is characterized by several wellknown neuropathological features, which result in a loss of synaptic activity. This dysfunction is readily reflected in regional decreases in cerebral metabolic activity. The characteristic topographic distribution of this hypometabolism, involving the associative cortex in the temporoparietal areas of both hemispheres and the posterior cingulate cortex and precuneus, is a distinctive feature of $\mathrm{AD}$, also in the earliest phase of the disease $[4,15,18,30]$. The primary motor, somatosensory, and visual cortical areas are relatively spared, whereas the frontolateral association cortex is frequently involved to a variable degree [15] (see Fig. 1).

Patients with $\mathrm{AD}$ display a multiplicity of cognitive deficits in domains such as memory, language, and attention, all of which can be linked to neurodysfunctional alterations detectable by FDG PET. The typical degenerative changes occur early in the disease course in the temporoparietal lobes, which mediate visuospatial attentional processing, and they are, in addition to the cardinal deficits in long-term memory, very pervasive in AD. FDG PET reports have supported this cognitive functional correlation [1]. Metabolic asymmetries 2 and associated predominance of language or visuospatial impairment are found in the early phase of $\mathrm{AD}$, and sometimes persist during its progression [36].

One of the largest FDG PET multicenter studies in the literature [15] showed the typical metabolic pattern in early $\mathrm{AD}$ and also a highly significant correlation between this initial metabolic impairment and subsequent decline of MMSE score during follow-up; this was particularly evident in mildly affected cases. Thus, impairment of glucose metabolism in the temporoparietal and frontal association cortex is not only an indicator of dementia severity, but also a predictor of the progression of cognitive symptoms. This pattern corresponds in general to the neuropsychological symptoms, characterized by impairment of memory and high-order cognition, including complex perceptual processing and planning of action. These changes differ from those of normal aging, which leads to predominantly medial frontal metabolic decline [37].

Early AD usually presents as a distinctive phenotype, characterized by a prevalent episodic memory impairment that is accompanied by specific neuropathological changes. According to neuropathological studies, the earliest pathological changes in AD develop in the transentorhinal and entorhinal regions. The neurofibrillary pathology then spreads into the hippocampus, and finally toward the neocortex [38]. Medial temporal metabolic reductions might thus be expected to be the earliest markers of the disease process related to the dominant cognitive impairment in the domain of long-term memory, but this has not generally 
Fig. 1 Regions that show significant reductions of FDG uptake (marked in red) in patients with probable $\mathrm{AD}$ in comparison with normal controls and correlated with dementia severity (MMSE scores). Regions with preserved FDG uptake are marked in blue. Slices are $2 \mathrm{~mm}$ thick and parallel to the AC-PC level. Modified from Herholz et al. [15] (color figure online)

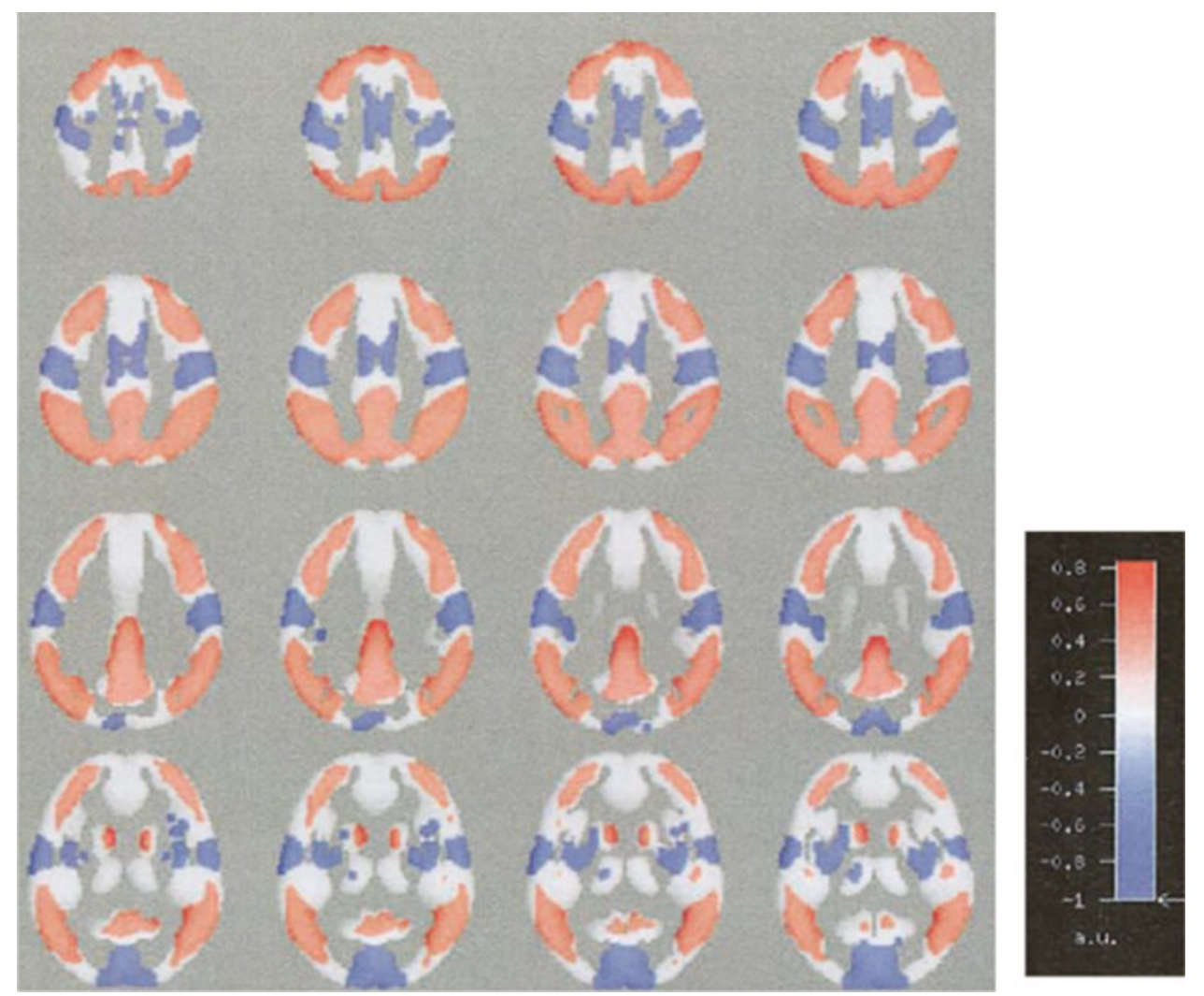

been found to be the case. First, the spatial resolution of PET systems is best in superficial cortical areas close to the detectors, while it is worst in midline and medial structures located far from the detectors. The normalization and smoothing procedures (part of SPM packages) that are necessary to minimize individual inhomogeneity in brain shape and dimensions may mask reduced uptake in small structures, such as the hippocampus. Therefore, it is difficult, with SPM, to identify hippocampal and, more in general, medial temporal lobe hypometabolism on FDG PET scans. Medial temporal hypometabolism is, instead, highlighted with ROI methods, which involve co-registration with MRI for accurate ROI positioning on the hippocampus on FDG PET scans, and metabolic reduction has indeed been observed in MCI and AD using this approach [30]. Finally, a pathophysiological explanation is that the high synaptic density at the posterior temporoparietal association cortex and limbic cortex makes it easier to detect glucose hypometabolism in these regions as compared to the medial temporal structures, which are rich in cell bodies but have relatively low synaptic density [39].

Villain and colleagues [40] have shown that the metabolic reduction in the cortex, including the posterior cingulate cortex and precuneus, is significantly correlated with hippocampal atrophy and cingulum bundle degeneration. Using structural MRI and $\left[{ }^{18} \mathrm{~F}\right]-\mathrm{FDG}$ PET in patients with early $\mathrm{AD}$, they assessed the relationships among hippocampal atrophy, white matter integrity and gray matter metabolism by means of a whole-brain voxel-based correlative approach. They found that hippocampal atrophy is specifically related to cingulum bundle disruption, which, in turn, is highly correlated with hypometabolism of the posterior cingulate cortex, but also of the middle cingulate gyrus, thalamus and mammillary bodies (all part of the Papez circuit), as well as the right temporoparietal associative cortex. These results provide direct evidence supporting the disconnection hypothesis as a major factor contributing to the early posterior hypometabolism in AD. Disruption of the cingulum bundle also relates to the hypometabolism in the connected network encompassing the whole Papez memory circuit. Notably, a correlation between glucose metabolism in the neocortex and entorhinal cortex, across both hemispheres, has also been shown in normal controls, whereas in $\mathrm{AD}$ patients this correlation is largely lost [41].

The key role of the posteromedial cortex (posterior cingulate and precuneus) in cognitive dysfunction assessed in $\mathrm{AD}$ is probably related to its highly integrated position within attentional, visuospatial and memory neural networks. Posterior cingulate metabolism was also found to be correlated with dementia severity, even when age was taken as a confounding covariate, whereas metabolism in the hippocampal formation was not shown to correlate with global cognitive deficit [42]. Higher posteromedial 
metabolic impairment in early-onset cases may reflect greater density of regional cerebral lesions or a major loss of functional afferences in a richly connected multimodal associative area [43].

In clinical practice, memory test performance may not distinguish between the dementia of AD and FTD, resulting in misdiagnosis. Specific patterns of cerebral hypometabolism in neurological patients are associated with different profiles of memory deficits [44]. In AD, exploration of the correlations between memory test scores and metabolic values across a sample of subjects provided a map of those brain structures whose synaptic dysfunction underlies particular neuropsychological alterations. The distribution of sites correlated with specific memory deficits showed striking differences depending on the memory system involved and the severity of the impairment [45]. A recent study using FDG PET set out to dissociate the neural correlates of memory disorders in AD and FTLD [46]. In $\mathrm{AD}$ patients, memory test performance was mainly correlated with FDG metabolic changes in the parieto-mesial (precuneus) cortex, whereas the performance of the FTLD patients was found to be correlated with changes in frontal cortical as well as subcortical regions. Thus, the neural correlates of memory problems differ almost completely in these two dementia syndromes, a finding clearly supported by the fact that memory functions are carried out by distributed networks which, as shown by FDG PET studies, break down with ongoing brain degeneration.

\section{Early cognitive decline: the prodromal AD phase}

Clinical diagnosis per se has limited accuracy in the early phase of $\mathrm{AD}$ and indeed requires the presence of precise cognitive symptoms; conversely, biomarkers specific for AD-related pathological phenomena would allow more accurate diagnosis in this phase. FDG PET studies have provided crucial evidence of functional and molecular changes in neurodegenerative diseases associated with cognitive decline [47]. FDG PET holds great promise for the early diagnostic assessment of subjects with $\mathrm{AD}$, given that it is not only a functional but also a topographical biomarker, and also that in a hypothetical cascade model of $\mathrm{AD}$ biomarkers, it precedes the onset of atrophy visible on MRI [48].

Mild cognitive impairment is an umbrella term widely used in clinical practice to identify a transitional condition between normal cognitive functioning and clinical dementia, usually of the AD subtype and also of the kind associated with other neurodegenerative conditions [49]. An individual with MCI is a non-demented person with objective cognitive deficits, either in single memory or non-memory domains, or in multiple cognitive domains, and a high risk of conversion to dementia (see [50] for a review). This clinical cognitive heterogeneity corresponds well to the variety of possible underlying neuropathological conditions that will progress to $\mathrm{AD}$ or other dementia subtypes; conversely, $30 \%$ of MCI subjects never develop dementia or $\mathrm{AD}$ [51].

Low uptake of $\left[{ }^{18} \mathrm{~F}\right]-\mathrm{FDG}$ in hippocampal structures is a typical feature of amnestic MCI (aMCI) patients' scans [41, 52], mostly related to hippocampal atrophy, thus much less pronounced after correction of partial volume effects. On the contrary, hypometabolism in the temporoparietal and posterior cingulate cortices is a more reliable predictor of progression to $\mathrm{AD}$ (23) and remains significant after partial volume correction [53]. It is worth noting that when the metabolic biomarker is negative on semi-quantitative assessment of FDG PET scans, used for single-subject analysis, the cognitive problems are unlikely to be attributable to $\mathrm{AD}$ or other dementia pathology [47].

Mosconi et al. [21], in a longitudinal clinical and FDG PET assessment of a case series of subjects with neuropathological diagnostic confirmation, showed brain metabolic profiles in life that were consistent with the postmortem diagnosis. This study also showed that glucose metabolic reductions precede the onset of clinical symptoms by several years and afterwards correlate with both dementia severity in life and pathological diagnosis of AD. Mapping the temporal course of the progressive involvement of different brain regions, glucose metabolic abnormalities were found to manifest themselves initially in the hippocampus, followed by the parietotemporal and posterior cingulate cortices at mild dementia stages (see, also, the contribution by Mosconi in the present issue, page XX).

A recent systematic and quantitative meta-analysis investigated the prototypical neural correlates of $\mathrm{AD}$ in its prodromal stage [4]. Only studies applying quantitative automated whole-brain analysis were included. Forty studies were identified, involving 1,351 patients and 1,097 healthy control subjects, reporting either atrophy or decreases in glucose utilization. The meta-analysis revealed that early $\mathrm{AD}$ affects the entorhinal and hippocampal regions structurally, and the inferior parietal lobules and precuneus functionally. The authors further suggested that atrophy in the entorhinal/hippocampus and hypometabolism/hypoperfusion in the inferior parietal lobules are the most reliable predictors of the progression from aMCI to $\mathrm{AD}$, whereas changes in the posterior cingulate cortex and precuneus are unspecific; in fully developed $\mathrm{AD}$, there was also involvement of a frontomedian-thalamic network [4].

Specific metabolic patterns and clinical/cognitive follow-up of the disease course confirmed that FDG PET imaging, particularly using SPM at single-subject level, is a reliable and accurate method for discriminating between 
different underlying dementia pathologies in prodromal AD [54].

Longitudinal studies on MCI patients provided evidence of different possible progressions, ranging from the development of $\mathrm{AD}$ or non-AD dementias, e.g. FTD or dementia with Lewy bodies (DLB), to the stabilization or even reversal of cognitive symptoms [55]. For example, the observation of specific metabolic reductions in the associative occipital cortex associated with visual perceptual disturbances and hallucinations suggests an underlying DLB pathology, and prefrontal medial and or dorsolateral hypometabolism in MCI patients predicts a diagnosis of FTD at clinical follow-up (see Figs. 2, 3, and 4).

Anchisi and colleagues [18] showed that neuropsychological testing alone can identify subjects who, having a relatively mild memory deficit, are not likely to progress to dementia, thus showing a high negative predictive value with regard to progression. However, prediction based on neuropsychological testing is less reliable for MCI patients with more severe memory impairment. In these patients, FDG PET adds significant information by separating those who will progress within the next 12 months from those who will remain stable.

The immediate diagnostic challenge-to accurately identify minimally affected patients early enough to allow them to reap the greatest potential therapeutic benefits- will be met best using the tools best equipped to facilitate the making of a highly sensitive and accurate diagnosis at the earliest stage of the disease. The current clinical and research studies support the use of FDG PET, as a means of obtaining more accurate evaluation of subjects with cognitive decline, in meeting this challenge.

\section{Atypical variants}

The core clinical symptom of AD is memory disturbance, but young subjects, in particular, often present with other cognitive deficits such as agnosia, apraxia or aphasia. This clinical heterogeneity has led to the recognition of posterior cortical atrophy (PCA) (visual), logopenic aphasia (language) and frontal (executive/behavioral) variants. These subtypes are characterized by metabolic dysfunction in specific brain regions, responsible for their clinical and cognitive features.

Posterior cortical atrophy is a clinico-radiological syndrome currently considered among the atypical presentations of AD. The progressive neurodegeneration affecting the parietal, occipital, and occipitotemporal cortices that underlies PCA is attributable to AD in most patients. There are, however, other possible underlying causes, including DLB, corticobasal degeneration, and prion disease, and not

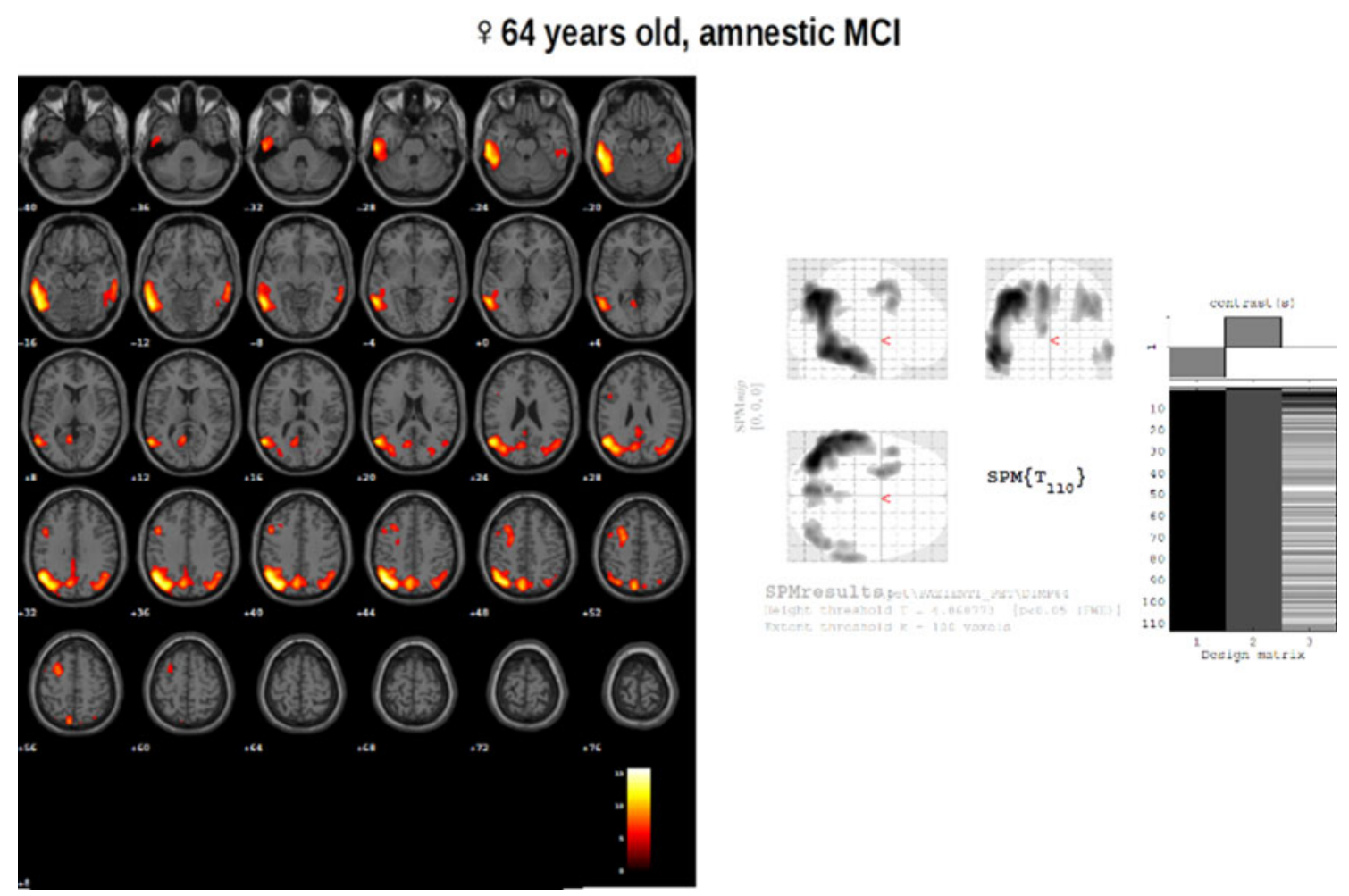

Fig. 2 Statistical parametric mapping: regions of significant decreases in FDG uptake, overlapped on the standard SPM template. The typical $\mathrm{AD}$ metabolic pattern suggests a diagnosis of prodromal $\mathrm{AD}$ 
all patients with PCA have atrophy on neuroradiological imaging [56].

Among the atypical dementias, this neurodegenerative syndrome is characterized by progressive decline in visuospatial, visuoperceptual, literacy, and praxic skills. Highorder visuospatial and visuoperceptual disorders as well as basic visual process impairment are core neuropsychological features. The combination of these symptoms reflects the involvement of distinct cortical areas and streams responsible for processing different types of visual information (i.e., the parietal or dorsal stream, the occipitotemporal or ventral stream, and the primary visual cortex). In addition, affected patients may present alexia, features of Balint and/or Gerstmann syndromes, and progressive oral language dysfunctions with prominent word retrieval difficulties, very reminiscent of a "logopenic syndrome" [56].

Data from functional imaging studies using FDG PET showed changes in occipitoparietal areas [57]. In addition to posterior regions, FDG PET has revealed specific areas of hypometabolism in the frontal eye fields bilaterally, which can occur secondary to loss of input from occipitoparietal regions and be the cause of oculomotor apraxia in PCA [58] (see Fig. 5).

The correct identification of PCA syndrome is a major challenge for clinicians, since its differential diagnosis is often very difficult on clinical grounds alone. Although neuroimaging studies have an important role to play in corroborating the clinical diagnosis, specific structural and/ or functional changes are not yet considered as supportive features in clinical practice. FDG PET imaging could disclose a crucial functional neural substrate of PCA that needs to be fully clarified. Greater awareness of the syndrome and agreement over the correspondence between syndrome-level and disease-level classifications are needed to improve diagnostic accuracy, clinical management, and the design of research studies. See also "Aphasia variants".

\section{Dementia with Lewy bodies}

From a clinical perspective, DLB presents as a progressive cognitive decline with disproportionate deficits of both visuospatial abilities and frontal executive function, accompanied by mild to moderate parkinsonism. Further accompanying features include spontaneous recurrent visual hallucinations and conspicuous fluctuations in alertness and cognitive performance [59]. The main differential diagnoses are with AD and Parkinson's disease dementia.

Neuroimaging, showing reduced occipital metabolism and perfusion and reduced striatal dopaminergic innervation, is increasingly used to supplement the clinical diagnosis of DLB [60].
Reduced FDG uptake is found to be very similar to what is observed in $\mathrm{AD}$, although it also extends to the primary and associative visual cortices, which are usually spared in $\mathrm{AD}$ (Fig. 4) [9, 61]. It is possible to observe functional asymmetry, with corresponding clinical features, namely more prevalent language or visuospatial dysfunction. In addition, at the same level of dementia severity, the global cerebral metabolic rate of glucose is lower in DLB than in $\mathrm{AD}$ [62]. The impairment of glucose metabolism in the visual cortex may well be the correlate of the impairment of visual processing and the visual hallucinations [63]. The medial temporal and posterior cingulate glucose metabolism is lower in AD than in DLB, possibly explaining the different memory profile and pointing to a different underlying pathology [64]. More specifically, in DLB [65], unlike $\mathrm{AD}$, hypometabolism in the lateral occipital cortex was found to show the highest sensitivity $(88 \%)$, but the relative preservation of the mid- or posterior cingulate gyrus (cingulate island sign) had the highest specificity $(100 \%)$. Thus, FDG PET can identify the hypometabolic patterns typical not only of $\mathrm{AD}$, but also of DLB dementia, associated with specific behavioral and cognitive impairments, and differentiate between the two $[9,65]$.

\section{Frontotemporal lobar degeneration (FTLD)}

FDG PET imaging is now considered a crucial element in the diagnostic workup of the FTLD spectrum, which includes different clinical variants.

An international consortium of researchers recently revised the current criteria in a bid to improve diagnostic accuracy for the behavioral variant [14]. Thus, a probable bvFTD diagnosis requires specific clinical features with evidence of progression and unequivocally positive FDG neuroimaging findings, while a definite diagnosis is made only in those cases with neuropathological confirmation or with a pathogenic gene mutation. The role of metabolic neuroimaging is crucial in the recent PPA classification, too. FDG PET functional data can make an important contribution to the diagnosis of different variants, because the site of maximal damage within the language network determines the different clinical presentations of PPA [8]

Diagnostic criteria for FTD also underline that alterations in personality and social conduct are a central clinical feature of the disease [66]. Because the primary brain areas affected in FTD are the frontal lobes, the anterior temporal lobes, and the amygdala [7], the observed personality changes ostensibly arise from a disruption of these structures' particular contribution to higher social functioning [67]. The sociopathic behavior manifested by some patients with FTD might reflect a combination of diminished emotional concern for the consequences of their actions 


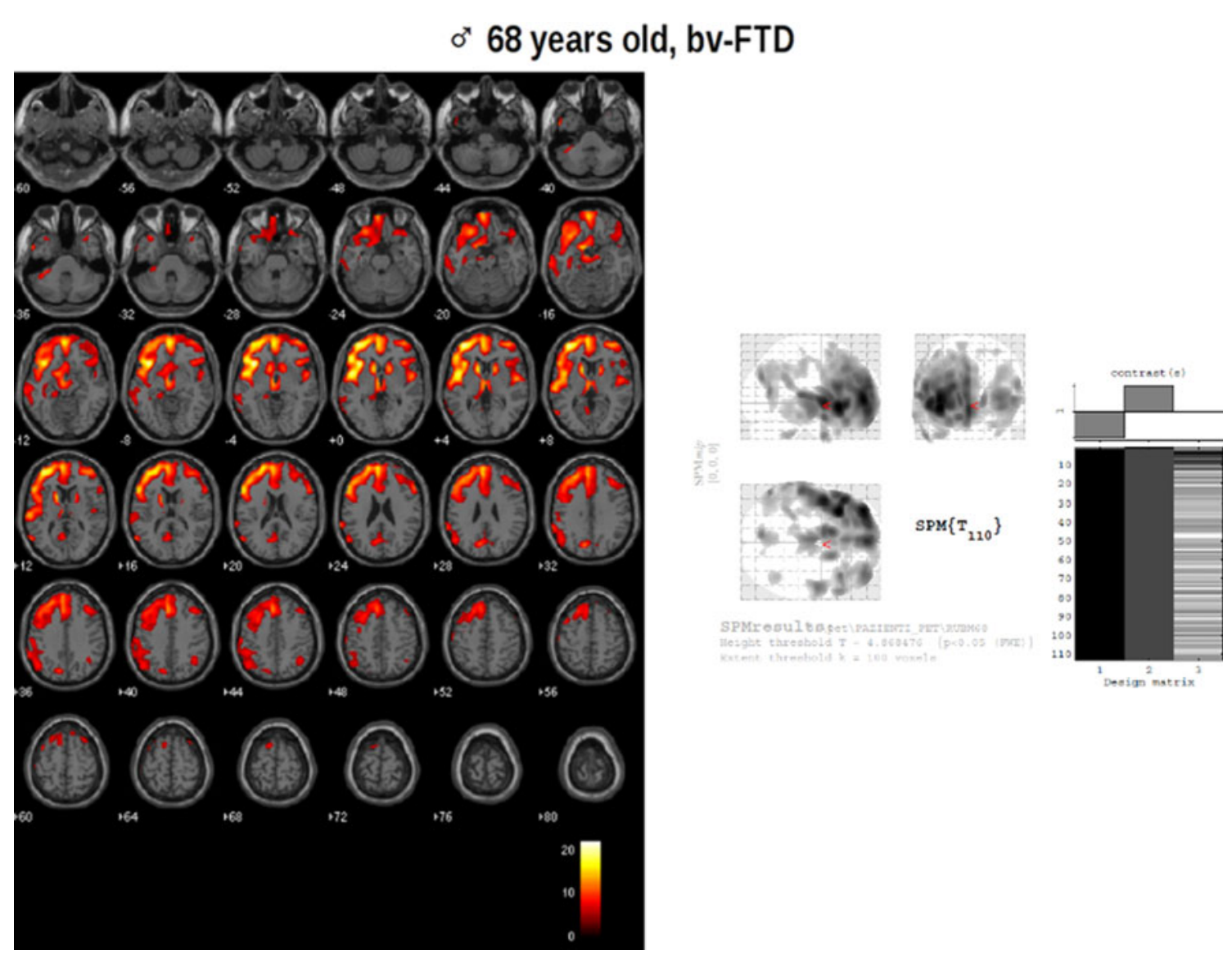

Fig. 3 Statistical parametric mapping: regions of significant decreases in FDG uptake, overlapped on the standard SPM template. The diagnosis is supported by the typical bvFTD metabolic pattern with involvement of the frontal medial and dorsolateral prefrontal cortex

and disinhibition, due to right frontotemporal dysfunction, evident on neuroimaging. Personality disorders can be thought of as trait-like dysfunctional patterns in impulse control. These domains of dysfunction have been linked to specific neural circuits [68].

\section{bvFTLD}

Patients with bvFTD present with insidious changes in personality and interpersonal conduct that indicate progressive disintegration of the neural circuits involved in social cognition, emotion regulation, motivation and decision making. The underlying pathological changes are heterogeneous and characterized by various intraneuronal inclusions. Supported by neuropsychological testing designed to detect impairment in decision making, emotion processing, and social cognition, FDG PET brain imaging can be considered the most sensitive diagnostic tool currently available [67].

Impairment of executive functions is a hallmark of the behavioral disorders associated with frontal type dementia. PET neuroimaging studies in the field also provided some evidence for the functional role of distinct prefrontal systems in different clinical aspects of bvFTD [6, 7, 69]. In a multicenter study, FDG PET images from population of patients with FTD were compared with images from controls of a similar age. A conjunction analysis led to identification of the ventromedial frontopolar cortex as the single region affected in each and every bvFTD patient. This regional metabolic impairment provides support for neuropsychological research data showing that the ventromedial frontal cortex is critically involved in decisionmaking processes based on personal experience, feelings of rightness, or social knowledge, processes that are characteristically impaired in bvFTD [6].

In patients with bvFTD, behavioral abnormalities may vary from apathy with motor slowness (apathetic form) to disinhibition with agitation (disinhibited form). These clinical presentations may be related to specific regional cerebral dysfunction [7]. The assessment of cerebral metabolism in patients fulfilling clinical criteria for bvFTLD showing, respectively, an apathetic or disinhibited behavioral syndrome revealed not only commonalities but also significant differences. A reduction of frontal glucose 
metabolism was present in the whole group of bvFTLD patients; however, the apathetic syndrome was associated with a prevalent dorsolateral and frontal medial hypometabolism, whereas the disinhibited syndrome with a selective hypometabolism in interconnected limbic structures (the cingulate cortex, hippocampus/amygdala, and accumbens nucleus) (Fig. 4). These FDG PET changes can be regarded as specific functional markers of the different behavioral presentations in bvFTLD.

FDG PET also has crucial implications with regard to the diagnostic criteria in bvFTD phenocopy syndrome. The diagnosis of bvFTD is by no means an easy task in the early stages of the disease, and many of the clinical symptoms overlap with those seen in psychiatric disorders, depression and other dementias. It is also increasingly apparent that a subset of patients who present with the clinical features of bvFTD does not progress to frank dementia. Such patients either remain stable over many years or improve. They show marginal impairment on neuropsychological tests of executive function, preserved memory and social cognition, and normal metabolic brain imaging. Crucially, these patients might present with depressive syndrome. Thus, FDG PET is particularly useful in helping to identify phenocopy cases as those who will show preserved frontal metabolism [69].
Aphasia variants

Several clinical variants have been described within the PPA spectrum [70]. Patients are classified into these variants on the basis of specific speech and language features, and the diagnosis may be further supported by neuroimaging if the expected pattern of atrophy and/or hypometabolism or hypoperfusion is found. Thus, the non-fluent/agrammatic variant (nfvPPA) is characterized by agrammatism and/or motor speech articulatory errors attributable to an apraxia of speech [71]. Neuroimagingsupported diagnostic markers are atrophy and/or functional abnormalities in the left posterior fronto-insular region $[8,72]$.

Semantic variant (svPPA) is considered a paradigmatic disorder of semantic memory, which can be associated with a characteristic anatomical profile on MRI characterized by asymmetric, selective antero-inferior temporal lobe atrophy. A pathophysiological model of semantic dementia is based on sequential, regionally determined disintegration of a vulnerable semantic neural network [73]. svPPA is a unique syndrome in the FTLD spectrum, typically presenting as progressive fluent anomic aphasia. The core features of svPPA are impaired confrontation naming and single-word comprehension. Object knowledge

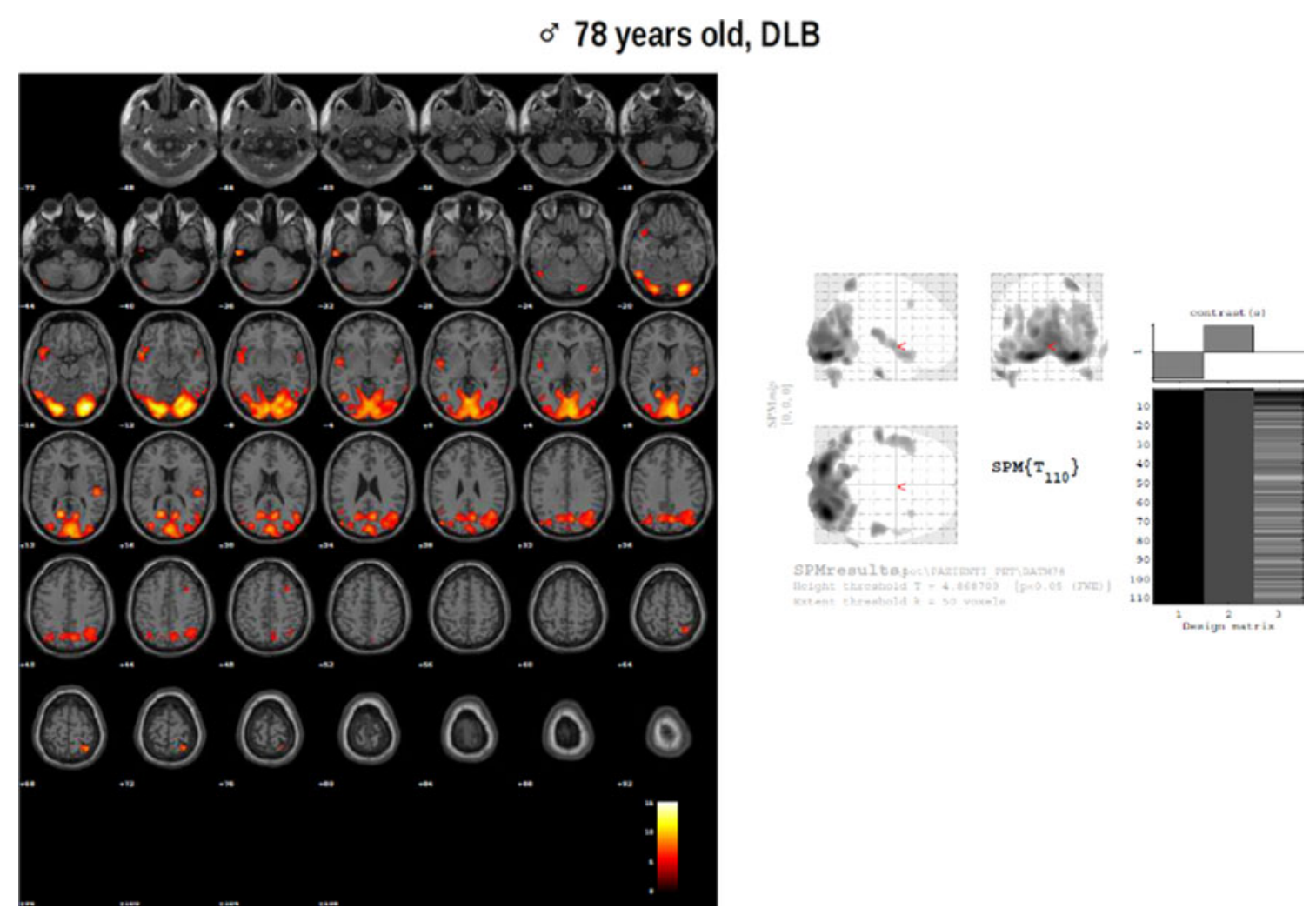

Fig. 4 Statistical parametric mapping: regions of significant decreases in FDG uptake, overlapped on the standard SPM template. The typical DLB metabolic pattern, with involvement of the occipital cortex, strongly favors a diagnosis of DLB 


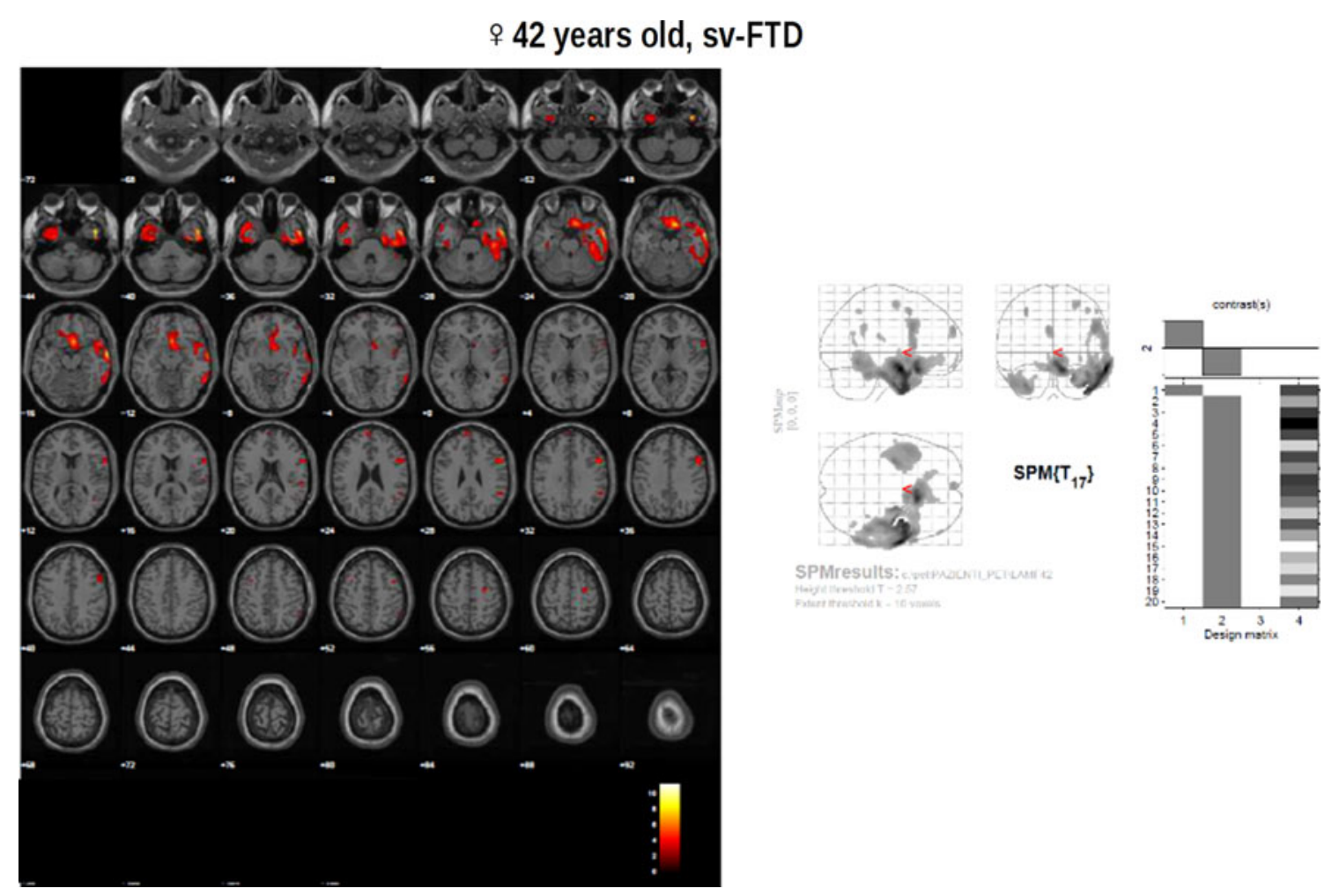

Fig. 5 Statistical parametric mapping: regions of significant decreases in FDG uptake, overlapped on the standard SPM template. The prevalent involvement of the anterior temporal cortex (temporal poles) supports a diagnosis of semantic dementia

is usually affected but repetition and motor speech are spared.

Cognitive features of the svPPA syndrome have been shown to correlate with the regional distribution of atrophy and metabolic dysfunction and, specifically, FDG PET showed verbal deficits especially associated with left dominant temporal lobe involvement, while non-verbal deficits, including prosopagnosia, other forms of object agnosia and impaired knowledge of living things, were linked to right temporal lobe involvement $[74,75]$.

The logopenic variant (lvPPA) [76] is defined by hesitant speech with word-finding pauses due to impaired single-word retrieval and difficulty in sentence repetition, although object knowledge and motor aspects of speech are spared. Consensus imaging-supported diagnostic markers are predominant left posterior perisylvian or parietal abnormalities [77].

Both structural and functional neuroimaging studies have established consistent neuroanatomical correlations in PPA variants at group level; however, the neuroimaging diagnostic criteria, especially those for nfvPPA and lvPPA, have not been widely validated at individual level in clinical practice [70, 78].

Systematic FDG PET studies using semi-quantitative/ voxel-based assessment in single individuals should be undertaken to provide features to support the diagnosis.

\section{Brain functional reserve}

The cognitive reserve theory argues that lifetime experience provides partial protection against the deleterious effects of aging and also against neurodegenerative pathology. Subjects with a high premorbid cognitive level can experience a lengthy period of cognitive functional decline before reaching the lower normal limit (dementia threshold) on standard neuropsychological tests.

Indeed, previous reports have shown a greater degree of impairment of regional cerebral metabolic activity for a given level of dementia in highly educated patients, a finding which suggests that these individuals have a functional reserve provided by their education, which masks the clinical expression of a higher degree of neurodegeneration.

Exploration of the neurobiological correlates of a higher educational and occupational level and the mechanisms by which a cognitive reserve may influence the expression of dementia have led to the hypothesis that a higher level of premorbid ability may, in general, allow for greater compensatory capacity in response to advancing disease, probably influencing brain function at the level of neuronal systems. There is substantial neuroimaging evidence of variability in brain function in healthy aging as well as in neurodegenerative diseases. This is usually interpreted as reflecting the recruitment of additional neural resources to 
support a normal level of performance, in the face of decreasing neural substrates. FDG PET studies have shown a significant association between higher levels of education/occupation and lower brain glucose metabolism in the posterior temporoparietal cortex and precuneus in $\mathrm{AD}$ and MCI converters, suggesting that education and occupation may be proxies for brain functional reserve, reducing the severity and delaying the clinical expression of $\mathrm{AD}$ pathology [25]. The results in aMCI converters suggest that the functional reserve is already at play in the pre-dementia phase of $\mathrm{AD}$ [25]. Correlations involved the posterior temporoparietal association cortices and precuneus, typically affected by $\mathrm{AD}$ pathology. These regions participate in cortical networks responsible for highly integrated tasks, including visuospatial imagery, episodic memory retrieval, and self-processing operations, as suggested by functional imaging studies in healthy controls $[79,80]$. These data, accounting for the influence of both education and occupation, further support the view that these two proxies of reserve act on a common brain substrate.

Comparable results have also been reported in individuals with $\mathrm{AD}$ and Apoe4 status [81], suggesting that lifestyle features may counteract the effect of this geneticbased risk factor.

Similarly, in FTD, as in AD, reserve mechanisms could work against the pathological process, as demonstrated by neuroimaging studies, where patients with higher reserve require a more severe extent of neuropathology to manifest the clinical symptomatology [26]. In the FTLD spectrum, behavioral disturbances such as apathy and disinhibition are closely related to damage of specific brain regions, as shown with FDG PET for selected brain networks [7]. In line with this concept, behavioral disturbances, and not only cognitive functions, might be modulated by reserve mechanisms [26].

The demonstration that cognitive reserve mechanisms are also at work in DLB, involving the brain regions affected by the disease, thus expands the concept of brain reserve to other neurodegenerative conditions [82].

\section{PET/MRI hybrid techniques}

The recently developed PET/MRI techniques are of particular interest for the fields of neurology and neuroscience, given that PET and MRI are the neuroimaging methods of choice for many clinical and scientific applications [32]. Crucial "technical factors" worth considering in relation to these techniques include a lower radiation exposure in comparison to CT/PET, the possibility of obtaining partial volume correction of PET data using MRI information, and the possibility of performing MRI-based assessment of blood-brain disruption for correlation with tracer uptake on PET.
In clinical routine, a single PET/MRI study session can be sufficient, in a patient with cognitive decline, to exclude vascular or neoplastic factors and obtain an accurate and quantified evaluation of brain atrophy (local or general) and of the specific metabolic patterns associated with neurodegenerative conditions. All this could improve the clinical diagnosis and the patient's comfort.

MRI brain volumetric measurements and PET metabolic and/or amyloid imaging are considered crucial tools for the assessment of disease biomarkers for early AD diagnosis and differential diagnosis versus other neurodegenerative dementias [10-14]. PET/MRI acquisition in a hybrid scanner may allow much more precise anatomical evaluation, on the basis of PET data, of disease state and neuronal injury. This is particularly important in the early phase of the disease when the amount of amyloid pathology and the metabolic dysfunction are still limited.

In the very frequent mixed forms of cognitive decline or dementia [83], an integrated MRI/PET approach will allow, in a single session, assessment of cerebrovascular changes and of the metabolic patterns of neuronal injury as the established sensitive and specific markers of neurodegeneration. The superior specificity of MRI for vascular lesions is, indeed, well known [84], as is the superior specificity of PET metabolic imaging for the accurate diagnosis of AD, LBD and FTD [9, 11, 14].

In study of the amyloid angiopathy, parallel evaluation of the presence or absence of the typical MRI signs (hemorrhages and residual brain lesions) and of the amyloid burden by PET will encourage co-interpretation of the two signals, adding further diagnostic and prognostic value [85]. The possible coexistence of amyloid angiopathy and AD neurodegeneration could be also better revealed by MRI/PET assessment.

In the research field, integrated PET/MRI acquisitions might have an important role to play in neuroscience for multi-parametric analysis of complex functions in neural networks. Indeed, the hybrid PET/MRI systems combine the excellent soft-tissue contrast at high resolution of MRI, together with its additional imaging options such as fMRI, diffusion- and perfusion-weighted imaging and spectroscopy, with the molecular and functional information provided by PET. In the study of neurodegenerative conditions, PET/MRI tools might allow the combination and integration of the molecular information (glucose metabolism, amyloid load, receptor and enzyme imaging) provided by PET imaging, with several morphological and functional parameters measured by MRI. PET neuroreceptor studies will benefit from detailed MRI anatomical information, in particular more on small neuronal nuclei.

In the near future, the application of PET/MRI techniques is destined to emerge as best approach to the in vivo study of the physiological and pathophysiological mechanisms underlying brain functioning and brain disorders. 


\section{Conclusions}

FDG PET neuroimaging has greatly influenced neurology and neuroscience research over the past two decades. The demonstration of specific brain functional derangements in different neurodegenerative diseases associated with specific behavioral and cognitive impairments has provided crucial answers on how human thinking, feeling, and cognition are instantiated in the brain. The methodological advances of 3D PET machines in image acquisition and reconstruction and the increasingly broad application of semi-quantitative or voxel-based methods for data analysis have been crucial in making FDG PET an important clinical and research tool in neurology and neuroscience.

In the field of neuropsychology and cognitive neuroscience, FDG PET has provided additional important insights into the cerebral organization of cognitive functions, such as memory and language, and the in vivo assessment of the functional modifications associated with normal aging and dementia. The high sensitivity and selectivity of FDG PET allow the probing of systems dysfunction at molecular level. Headway is now being made at the level of early diagnosis and differential diagnosis of dementia conditions. Brain glucose metabolism with FDG PET has become the fundamental biomarker for neuronal injury, included in the recent clinical and research guidelines. The implications of these possibilities for the assessment of the effectiveness of therapeutic interventions in the field of normal and pathological aging are now starting to become clear, and remarkable progress may be expected in the coming years. This progress will depend on the integration of behavioral, neuropsychological and computational approaches with neuroimaging, and also on the integration of spatial, namely MRI and fMRI, data with molecular PET data, using the new hybrid machines. Mapping each functional signal anatomically and exploring its relationship with its molecular basis in a common framework will open the way for new discoveries, predictions and insights into neuroscience and neurology.

Conflict of interest Daniela Perani declares no conflict of interest.

Human and Animal Studies This article does not contain any studies with human or animal subjects performed by the author.

\section{References}

1. Perani D (2008) Functional neuroimaging of cognition. Handbook of Clinical Neurology, vol. 88 (3rd series). In: Goldenberg G, Miller B (eds) Neuropsychology and behavioral neurology. Elsevier, Philadelphia, pp 61-111
2. Price CJ (2012) A review and synthesis of the first 20 years of PET and fMRI studies of heard speech, spoken language and reading. Neuroimage 62:816-847

3. Magistretti PJ (2000) Cellular bases of functional brain imaging: insights from neuron-glia metabolic coupling. Brain Res 886:108-112

4. Schroeter ML, Stein T, Maslowski N, Neumann J (2009) Neural correlates of Alzheimer's disease and mild cognitive impairment: a systematic and quantitative meta-analysis involving 1351 patients. Neuroimage 47:1196-1206

5. Rabinovici GD, Miller BL (2010) Frontotemporal lobar degeneration: epidemiology, pathophysiology, diagnosis and management. CNS Drugs 24:375-398

6. Salmon E, Garraux G, Delbeuck X, Collette F, Kalbe E, Zuendorf G, Perani D, Fazio F, Herholz K (2003) Predominant ventromedial frontopolar metabolic impairment in frontotemporal dementia. Neuroimage 20:435-440

7. Franceschi M, Anchisi D, Pelati O, Zuffi M, Matarrese M, Moresco RM et al (2005) Glucose metabolism and serotonin receptors in the frontotemporal lobe degeneration. Ann Neurol $57: 216-225$

8. Josephs KA, Duffy JR, Fossett TR, Strand EA, Claassen DO, Whitwell JL et al (2010) Fluorodeoxyglucose F18 positron emission tomography in progressive apraxia of speech and primary progressive aphasia variants. Arch Neurol 67:596-605

9. McKeith IG, Dickson DW, Lowe J, Emre M, O’Brien JT, Feldman $\mathrm{H}$ et al (2005) Diagnosis and management of dementia with Lewy bodies: third report of the DLB consortium. Neurology 65:1863-1872

10. Dubois B, Feldman HH, Jacova C, Cummings JL, DeKosky ST, Barberger-Gateau P et al (2010) Revising the definition of Alzheimer's disease: a new lexicon. Lancet Neurol 9:1118-1127

11. McKhann GM, Knopman DS, Chertkow H, Hyman BT, Jack CR Jr, Kawas CH, Klunk WE, Koroshetz WJ, Manly JJ, Mayeux R, Mohs RC, Morris JC, Rossor MN, Scheltens P, Carrillo MC, Thies B, Weintraub S, Phelps CH (2011) The diagnosis of dementia due to Alzheimer's disease: recommendations from the National Institute on Aging-Alzheimer's Association workgroups on diagnostic guidelines for Alzheimer's disease. Alzheimers Dement 7:263-269

12. Albert MS, DeKosky ST, Dickson D, Dubois B, Feldman HH, Fox NC et al (2011) The diagnosis of mild cognitive impairment due to Alzheimer's disease: recommendations from the National Institute on Aging-Alzheimer's Association workgroups on diagnostic guidelines for Alzheimer's disease. Alzheimers Dement 7:270-279

13. Sperling RA, Aisen PS, Beckett LA, Bennett DA, Craft S, Fagan AM et al (2011) Toward defining the preclinical stages of Alzheimer's disease: recommendations from the National Institute on Aging-Alzheimer's Association workgroups on diagnostic guidelines for Alzheimer's disease. Alzheimers Dement 7:280-292

14. Rascovsky K, Hodges JR, Knopman D, Mendez MF, Kramer JH, Neuhaus J et al (2011) Sensitivity of revised diagnostic criteria for the behavioural variant of frontotemporal dementia. Brain 134:2456-2477

15. Herholz K, Salmon E, Perani D, Baron JC, Holthoff V, Frölich L et al (2002) Discrimination between Alzheimer dementia and controls by automated analysis of multicenter FDG PET. Neuroimage 17:302-316

16. Jeong Y, Cho SS, Park JM, Kang SJ, Lee JS, Kang E et al (2005) 18F-FDG PET findings in frontotemporal dementia: an SPM analysis of 29 patients. J Nucl Med 46:233-239

17. Foster NL, Heidebrink JL, Clark CM, Jagust WJ, Arnold SE, Barbas NR et al (2007) FDG-PET improves accuracy in 
distinguishing frontotemporal dementia and Alzheimer's disease. Brain 130:2616-2635

18. Anchisi D, Borroni B, Franceschi M, Kerrouche N, Kalbe E, Beuthien-Beumann B et al (2005) Heterogeneity of brain glucose metabolism in mild cognitive impairment and clinical progression to Alzheimer disease. Arch Neurol 62:1728-1733

19. Mosconi L, Tsui WH, Herholz K, Pupi A, Drzezga A, Lucignani $\mathrm{G}$ et al (2008) Multicenter standardized 18F-FDG PET diagnosis of mild cognitive impairment, Alzheimer's disease, and other dementias. J Nucl Med 49:390-398

20. Herholz K (2010) Cerebral glucose metabolism in preclinical and prodromal Alzheimer's disease. Expert Rev Neurother 10:16671673

21. Mosconi L, Mistur R, Switalski R, Tsui WH, Glodzik L, Li Y et al (2009) FDG-PET changes in brain glucose metabolism from normal cognition to pathologically verified Alzheimer's disease. Eur J Nucl Med Mol Imaging 36:811-822

22. Juh R, Pae CU, Kim TS, Lee CU, Choe B, Suh T (2005) Cerebral glucose metabolism in corticobasal degeneration comparison with progressive supranuclear palsy using statistical mapping analysis. Neurosci Lett 383:22-27

23. Hellwig S, Amtage F, Kreft A, Buchert R, Winz OH, Vach W et al (2012) $\left.{ }^{[18} \mathrm{F}\right]$ FDG-PET is superior to $\left[{ }^{123} \mathrm{I}\right]$ IBZM-SPECT for the differential diagnosis of parkinsonism. Neurology 79:13141322

24. Perneczky R, Drzezga A, Diehl-Schmid J et al (2006) Schooling mediates brain reserve in Alzheimer's disease: findings of fluorodeoxy-glucose-positron emission tomography. J Neurol Neurosurg Psychiatry 77:1060-1063

25. Garibotto V, Borroni B, Kalbe E, Herholz K, Salmon E, Holtoff $\mathrm{V}$ et al (2008) Education and occupation as proxies for reserve in aMCI converters and AD: FDG-PET evidence. Neurology 71:1342-1349

26. Spreng RN, Drzezga A, Diehl-Schmid J, Kurz A, Levine B, Perneczky R (2011) Relationship between occupation attributes and brain metabolism in frontotemporal dementia. Neuropsychologia 49:3699-3703

27. Minoshima S, Frey KA, Koeppe RA et al (1995) A diagnostic approach in Alzheimer's disease using three-dimensional stereotactic surface projections of fluorine-18-FDG PET. J Nucl Med 36:1238-1248

28. Signorini M, Paulesu E, Friston K et al (1999) Rapid assessment of regional cerebral metabolic abnormalities in single subjects with quantitative and nonquantitative [18F]FDG PET: a clinical validation of statistical parametric mapping. Neuroimage 9:63-80

29. Caroli A, Prestia A, Chen K, Ayutyanont N, Landau SM, Madison CM, Haense C, Herholz K, Nobili F, Reiman EM, Jagust WJ, Frisoni GB, EADC-PET Consortium, NEST-DD, and Alzheimer's Disease Neuroimaging Initiative (2012) Summary metrics to assess Alzheimer disease-related hypometabolic pattern with 18F-FDG PET: head-to-head comparison. J Nucl Med 53:592-600

30. Mosconi L (2005) Brain glucose metabolism in the early and specific diagnosis of Alzheimer's disease: FDG-PET studies in MCI and AD. Eur J Nucl Med Mol Imaging 32:486-510

31. Patterson JC, Lilien DL, Takalkar A, Pinkston JB (2011) Early detection of brain pathology suggestive of early $\mathrm{AD}$ using objective evaluation of FDG-PET scans. Int J Alzheimers Dis. doi:10.4061/2011/946590

32. Garibotto V, Forster S, Haller S, Vargas MI, Drzezga A (2013) Molecular neuroimaging with PET/MRI. Clin Transl Imaging 1:53-64

33. Teune LK, Bartels AL, De Jong BM, Willemsen ATM, Eshuis SA, De Vries JJ et al (2010) Typical cerebral metabolic patterns in neurodegenerative brain diseases. Mov Disord 25:2395-2404
34. Della Rosa P, Cerami C, Prestia A et al (2012) Clinical validation of a grid-based SPM web tool for the automatic assessment of $\left[{ }^{18}\right.$ F]FDG PET brain metabolic abnormalities in single subjects (P03.106). Neurology 78(Abstracts 1):P03.106

35. Landau SM, Harvey D, Madison CM et al (2011) Associations between cognitive, functional, and FDG-PET measures of decline in AD and MCI. Neurobiol Aging 32:1207-1218

36. Haxby JV, Grady CL, Koss E, Horwitz B, Heston L, Schapiro M et al (1990) Longitudinal study of cerebral metabolic asymmetries and associated neurophysiological patterns in early dementia of the Alzheimer type. Arch Neurol 47:753-760

37. Chételat G, Landeau B, Salmon E, Yakushev I, Bahri MA, Mézenge F et al (2013) Relationships between brain metabolism decrease in normal aging and changes in structural and functional connectivity. Neuroimage 76:167-177

38. Braak H, Braak E (1991) Neuropathological staging of Alzheimer-related changes. Acta Neuropathol 82:239-259

39. Morbelli S, Piccardo A, Villavecchia G, Dessi B, Brugnolo A, Piccini A, Caroli A, Frisoni G, Rodriguez G, Nobili F (2010) Mapping brain morphological and functional conversion patterns in amnestic MCI: a voxel-based MRI and FDG-PET study. Eur J Nucl Med Mol Imaging 37:36-45

40. Villain N, Desgranges B, Viader F, de la Sayette V, Mézenge F, Landeau B, Baron JC, Eustache F, Chételat G (2008) Relationships between hippocampal atrophy, white matter disruption, and gray matter hypometabolism in Alzheimer's disease. J Neurosci 28:6174-6181

41. Mosconi L, Tsui WH, De Santi S et al (2005) Reduced hippocampal metabolism in MCI and AD: automated FDG-PET image analysis. Neurology 64:1860-1867

42. Salmon E, Collette F, Degueldre C, Lemaire C, Franck G (2000) Voxel-based analysis of confounding effects of age and dementia severity on cerebral metabolism in Alzheimer's disease. Hum Brain Mapp 10:39-48

43. Hirao K, Ohnishi T, Matsuda H, Nemoto K, Hirata Y, Yamashita $\mathrm{F}$ et al (2006) Functional interactions between entorhinal cortex and posterior cingulate cortex at the very early stage of Alzheimer's disease using brain perfusion single-photon emission computed tomography. Nucl Med Commun 27:151-156

44. Perani D, Bressi S, Cappa SF et al (1993) Evidence of multiple memory systems in the human brain. A [18F] FDG PET metabolic study. Brain 116:903-919

45. Desgranges B, Baron J-, Lalevée C, Giffard B, Viader F, De La Sayette V et al (2002) The neural substrates of episodic memory impairment in Alzheimer's disease as revealed by FDG-PET: relationship to degree of deterioration. Brain 125:1116-1124

46. Frisch S, Dukart J, Vogt B, Horstmann A, Becker G, Villringer A et al (2013) Dissociating memory networks in early Alzheimer's disease and frontotemporal lobar degeneration-a combined study of hypometabolism and atrophy. PLoS One 8:e55251. doi:10.1371/journal.pone.0055251

47. Jagust W, Reed B, Mungas D, Ellis W, DeCarli C (2007) What does fluorodeoxyglucose PET imaging add to a clinical diagnosis of dementia? Neurology 69:871-877

48. Jack CR Jr, Knopman DS, Jagust WJ, Petersen RC, Weiner MW, Aisen PS, Shaw LM, Vemuri P, Wiste HJ, Weigand SD, Lesnick TG, Pankratz VS, Donohue MC, Trojanowski JQ (2013) Tracking pathophysiological processes in Alzheimer's disease: an updated hypothetical model of dynamic biomarkers. Lancet Neurol 12:207-216

49. Petersen RC, Doody R, Kurz A, Mohs RC, Morris JC, Rabins PV, Ritchie K, Rossor M, Thal L, Winblad B (2001) Current concepts in mild cognitive impairment. Arch Neurol 58:1985-1992

50. Gainotti G (2010) Origins, controversies and recent developments of the MCI construct. Curr Alzheimer Res 7:271-279 
51. Gabryelewicz T, Styczynska M, Luczywek E, Barczak A, Pfeffer A, Androsiuk W, Chodakowska-Zebrowska M, Wasiak B, Peplonska B, Barcikowska M (2007) The rate of conversion of mild cognitive impairment to dementia: predictive role of depression. Int J Geriatr Psychiatry 22:563-567

52. Clerici F, Del Sole A, Chiti A et al (2009) Differences in hippocampal metabolism between amnestic and non-amnestic MCI subjects: automated FDG-PET image analysis. Q J Nucl Med Mol Imaging 53:646-657

53. Jauhiainen AM, Kangasmaa T, Rusanen M et al (2008) Differential hypometabolism patterns according to mild cognitive impairment subtypes. Dement Geriatr Cogn Disord 26:490498

54. Yuan Y, Gu ZX, Wei WS (2009) Fluorodeoxyglucose-positronemission tomography, single-photon emission tomography, and structural MR imaging for prediction of rapid conversion to Alzheimer disease in patients with mild cognitive impairment: a meta-analysis. AJNR Am J Neuroradiol 30:404-410

55. Silverman DH, Truong CT, Kim SK et al (2003) Prognostic value of regional cerebral metabolism in patients undergoing dementia evaluation: comparison to a quantifying parameter of subsequent cognitive performance and to prognostic assessment without PET. Mol Genet Metab 80:350-355

56. Crutch SJ, Lehmann M, Schott JM, Rabinovici GD, Rossor MN, Fox NC (2012) Posterior cortical atrophy. Lancet Neurol $11: 170-178$

57. Kas A, de Souza LC, Samri D et al (2011) Neural correlates of cognitive impairment in posterior cortical atrophy. Brain 134:1464-1478

58. Nestor PJ, Caine D, Fryer TD, Clarke J, Hodges JR (2003) The topography of metabolic deficits in posterior cortical atrophy (the visual variant of Alzheimer's disease) with FDG-PET. J Neurol Neurosurg Psychiatry 74:1521-1529

59. Verghese J, Crystal HA, Dickson DW, Lipton RB (1999) Validity of clinical criteria for the diagnosis of dementia with Lewy bodies. Neurology 53:1974-1982

60. Garibotto V, Montandon ML, Viaud CT, Allaoua M, Assal F, Burkhard PR et al (2013) Regions of interest-based discriminant analysis of DaTSCAN SPECT and FDG-PET for the classification of dementia. Clin Nucl Med 38:e112-e117

61. Minoshima S, Foster NL, Petrie EC, Albin RL, Frey KA, Kuhl DE (2002) Neuroimaging in dementia with Lewy bodies: metabolism, neurochemistry, and morphology. J Geriatr Psychiatry Neurol 15:200-209

62. Ishii K, Imamura T, Sasaki M, Yamaji S, Sakamoto S, Kitagaki H, Hashimoto M, Hirono N, Shimomura T, Mori E (1998) Regional cerebral glucose metabolism in dementia with Lewy bodies and Alzheimer's disease. Neurology 51:125-130

63. Imamura T, Ishii K, Hirono $\mathrm{N}$ et al (1999) Visual hallucinations and regional cerebral metabolism in dementia with Lewy bodies (DLB). Neuroreport 10:1903-1907

64. Imamura T, Ishii K, Sasaki M et al (1997) Regional cerebral glucose metabolism in dementia with Lewy bodies and Alzheimer's disease: a comparative study using positron emission tomography. Neurosci Lett 235:49-52

65. Lim SM, Katsifis A, Villemagne VL, Best R, Jones G, Saling M, Bradshaw J, Merory J, Woodward M, Hopwood M, Rowe CC (2009) The 18F-FDG PET cingulate island sign and comparison to 123I-beta-CIT SPECT for diagnosis of dementia with Lewy bodies. J Nucl Med 50:1638-1645

66. Neary D, Snowden JS, Gustafson L et al (1998) Frontotemporal lobar degeneration: a consensus on clinical diagnostic criteria. Neurology 51:1546-1554

67. Piguet O, Hornberger M, Mioshi E, Hodges JR (2011) Behavioural-variant frontotemporal dementia: diagnosis, clinical staging, and management. Lancet Neurol 10:162-172
68. McCloskey MS, Phan KL, Coccaro EF (2005) Neuroimaging and personality disorders. Curr Psychiatry Rep 7:65-72

69. Kipps CM, Hodges JR, Fryer TD, Nestor PJ (2009) Combined magnetic resonance imaging and positron emission tomography brain imaging in behavioural variant frontotemporal degeneration: refining the clinical phenotype. Brain 132:2566-2578

70. Gorno-Tempini ML, Hillis AE, Weintraub S, Kertesz A, Mendez M, Cappa SF, Ogar JM, Rohrer JD, Black S, Boeve BF, Manes F, Dronkers NF, Vandenberghe R, Rascovsky K, Patterson K, Miller BL, Knopman DS, Hodges JR, Mesulam MM, Grossman M (2011) Classification of primary progressive aphasia and its variants. Neurology 76:1006-1014

71. Grossman M (2012) The non-fluent/agrammatic variant of primary progressive aphasia. Lancet Neurol 11:545-555

72. Rabinovici GD, Jagust WJ, Furst AJ, Ogar JM, Racine CA, Mormino EC, O'Neil JP, Lal RA, Dronkers NF, Miller BL, Gorno-Tempini ML (2008) A beta amyloid and glucose metabolism in three variants of primary progressive aphasia. Ann Neurol 64:388-401

73. Hodges JR, Patterson K, Oxbury S, Funnell E (1992) Semantic dementia. Progressive fluent aphasia with temporal lobe atrophy. Brain 115:1783-1806

74. Desgranges B, Matuszewski V, Piolino P, Chételat G, Mézenge F, Landeau B, de la Sayette V et al (2007) Anatomical and functional alterations in semantic dementia: a voxel-based MRI and PET study. Neurobiol Aging 28:1904-1913

75. Drzezga A, Grimmer T, Henriksen G, Stangier I, Perneczky R, Diehl-Schmid J, Mathis CA et al (2008) Imaging of amyloid plaques and cerebral glucose metabolism in semantic dementia and Alzheimer's disease. Neuroimage 39:619-633

76. Gorno-Tempini ML, Dronkers NF, Rankin KP, Ogar JM, La Phengrasamy BA, Rosen HJ, Johnson JK, Weiner MW, Miller BL (2004) Cognition and anatomy in three variants of primary progressive aphasia. Ann Neurol 55:335-346

77. Madhavan A, Whitwell JL, Weigand SD, Duffy JR, Strand EA, Machulda MM, Tosakulwong N, Senjem ML, Gunter JL, Lowe VJ, Petersen RC, Jack CR Jr, Josephs KA (2013) FDG PET and MRI in logopenic primary progressive aphasia versus dementia of the Alzheimer's type. PLoS One 8:e62471

78. Gil-Navarro S, Lladó A, Rami L, Castellví M, Bosch B, Bargalló $\mathrm{N}$ et al (2013) Neuroimaging and biochemical markers in the three variants of primary progressive aphasia. Dement Geriatr Cogn Disord 35:106-117

79. Cavanna AE, Trimble MR (2006) The precuneus: a review of its functional anatomy and behavioural correlates. Brain 129:564-583

80. Morbelli S, Perneczky R, Drzezga A, Frisoni GB, Caroli A, Van Berckel BNM et al (2013) Metabolic networks underlying cognitive reserve in prodromal Alzheimer disease: a European Alzheimer disease consortium project. J Nucl Med 54:894-902

81. Garibotto V, Borroni B, Sorbi S, Cappa SF, Padovani A, Perani D (2012) Education and occupation provide reserve in both ApoE $\varepsilon 4$ carrier and noncarrier patients with probable Alzheimer's disease. Neurol Sci 33:1037-1042

82. Perneczky R, Häussermann P, Drzezga A, Boecker H, Granert O, Feurer R, Förstl H, Kurz A (2009) Fluoro-deoxy-glucose positron emission tomography correlates of impaired activities of daily living in dementia with Lewy bodies: implications for cognitive reserve. Am J Geriatr Psychiatry 17:188-195

83. Rönnemaa E, Zethelius B, Lannfelt L, Kilander L (2011) Vascular risk factors and dementia: 40-year follow-up of a population-based cohort. Dement Geriatr Cogn Disord 31:460-466

84. Jadhav AP, Jovin TG (2012) Vascular imaging of the head and neck. Semin Neurol 32:401-410

85. Gurol ME, Dierksen G, Betensky R et al (2012) Predicting sites of new hemorrhage with amyloid imaging in cerebral amyloid angiopathy. Neurology 79:320-326 